\title{
Envirobot: A Bio-Inspired Environmental Monitoring Platform
}

\author{
Behzad Bayat, Alessandro Crespi, and Auke Ijspeert \\ Biorobotics laboratory (BioRob) \\ Ecole polytechnique fédérale de Lausanne (EPFL) \\ 1015 Lausanne, Switzerland \\ \{behzad.bayat, alessandro.crespi, auke.ijspeert\}@epfl.ch
}

\begin{abstract}
Autonomous marine vehicles are becoming essential tools in aquatic environmental monitoring systems, and can be used for instance for data acquisition, remote sensing, and mapping of the spatial extent of pollutant spills. In this work, we present an unconventional bio-inspired autonomous robot aimed for execution of such tasks. The Envirobot platform is based on our existing segmented anguilliform swimming robots, but with important adaptations in terms of energy use and efficiency, control, navigation, and communication possibilities. To this end, Envirobot has been designed to have more endurance, flexible computational power, long range communication link, and versatile flexible environmental sensor integration. Its low level control is powered by an ARM processor in the head unit and micro processors in each active module. On top of this, integration of a computer-on-module enables versatile high level control methods. We present some preliminary results and experiments done with Envirobot to test the added navigation and control strategies.
\end{abstract}

Index Terms-Autonomous marine vehicle, anguilliform swimming robot, environmental monitoring.

\section{INTRODUCTION}

In the recent decades, the marine robotic community has had an increasing interest to build vehicles for ocean exploration and exploitation. Remotely operated vehicles (ROV), autonomous surface/underwater vehicles (ASV/AUV), and gliders are fruitful products of these efforts. The vast majority of these underwater robots are propeller-driven, however, the efficiency of the currently adopted rotary propellers can hardly reach even half of the propulsion efficiency of fish, that is about $90 \%$ [1].

The idea of building a robotic fish that is as efficient, agile, and quiet as a natural fish is still far from becoming a reality. As stated by the authors in [1], in the next 10 years the possibility of a robot fish which will be able to swim with real fish in open water will get much more likely. Nevertheless, many research groups have studied aquatic locomotion and its implementation on a fish like robot. Including, the first robot fish from MIT [2], the eel REEL robots mimicking anguilliform Locomotion [3], the lamprey like robot which is controlled through a neuromuscular interface [4], a biorobotic platform inspired by the lamprey [5], the underwater snake robot Mamba developed at NTNU which propels using both aquatic locomotion and conventional propellers [6], and many more reported in [1].

This work was supported by Envirobot, a project of the Swiss NanoTera program, Natural Sciences.

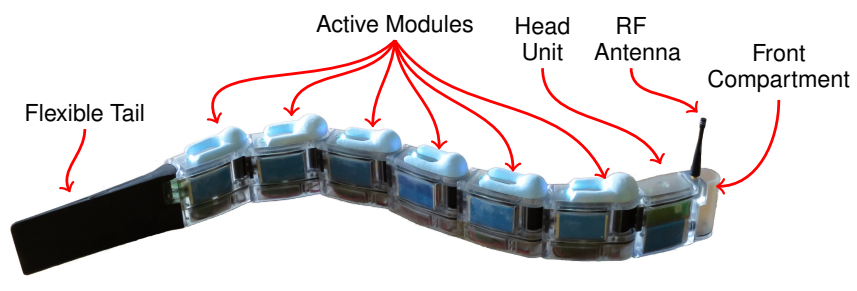

Fig. 1. Envirobot with six active modules, flexible tail, RF antenna, front compartment, and the head unit.

Taking inspiration from snakes and elongate fishes such as lampreys, Envirobot is an amphibious robot for outdoor robotic tasks. Its design is based on existing segmented anguilliform swimming robots, AmphiBot I [7], AmphiBot II [8], and AmphiBot III [9]. One of the main goals of the Envirobot project [10] is to design and construct an aquatic water sampling and water analysis robot, which can either work in a surveying mode according to a predefined path, or in autonomous-navigation mode, according to chemosensory and input from biological sensors; and that can store and/or communicate data analysis to an external observer. In autonomous surveying mode the robot will sample and analyze waterbodies according to a predefined path and number of waypoints. During autonomous-navigation mode, the robot must guide its movements and sampling on the basis of the sensory input. Autonomous-navigation is challenging but extremely useful, since Envirobot would be able to track and follow gradients of sparsely measured chemical pollution in waterbodies to find the source of pollution [11], [12].

The organization of the paper is as follows. Hardware architecture of the platform is introduced in section II. Section III is dedicated to software architecture and information flow in Envirobot. Some preliminary results are presented in section IV. The paper concludes in section $\mathrm{V}$ with conclusions and future directions.

\section{HARDWARE ARChitecture}

The design of Envirobot is inspired by aquatic locomotion of elongate fishes like lamprey and eel to mimic anguilliform swimming. Eels in particular are known to be efficient swimmers, which can cover long distances of about 5000 kilometers in a migration lasting for about 180 days [13]. 


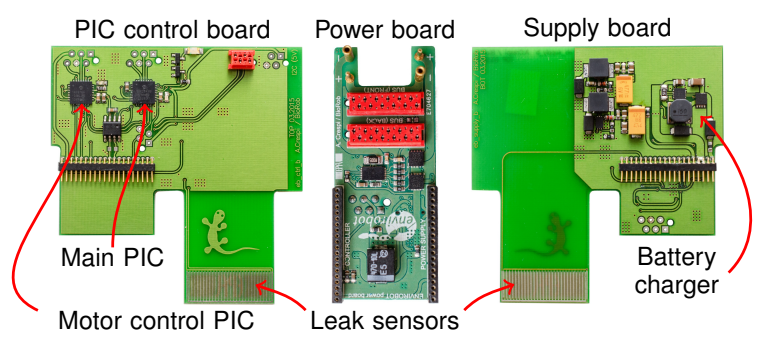

Fig. 2. Custom electronic boards of Envirobot active modules. From left to right: active modules control board, power board, and supply board.
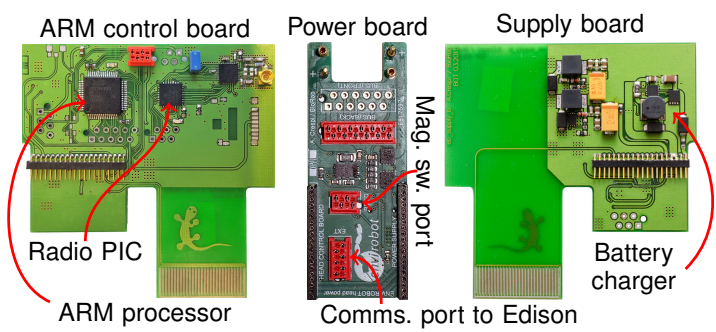

Fig. 3. Custom electronic boards of Envirobot head unit. From left to right: head unit control board, power board, and supply board.

Following the design principles of AmphiBot I-III, Envirobot is composed of a set of independent active modules, each with one degree of freedom that controls the angle (in the horizontal plane) between the module and the one connected in the front. By controlling the relative angle at each joint (between each consecutive module), it is possible to generate a traveling wave of body undulation along the body of Envirobot. As a consequence, a backward traveling wave will propel the robot forward. Each module is designed such that it is slightly buoyant, which results in keeping the robot at the water surface. Furthermore, each module has dimensions of $125 \mathrm{~mm} \times 50 \mathrm{~mm} \times 90 \mathrm{~mm}$ in length, width, and height, respectively. Since tail plays a very important role in providing thrust in anguilliform swimming [14], a flexible tail with a length of $240 \mathrm{~mm}$ is attached to the very last module.

In the current design architecture, the traveling wave setpoints for the joints angles of active modules are generated via a centralized controller located in the un-actuated head module. Due to its modularity, the number of active modules can be increased or decreased depending on the application and availability of the modules. Fig. 1 shows Envirobot with 6 active modules, a passive flexible tail to provide more thrust, an un-actuated head module which carries an RF antenna for remote command and control, and the front compartment that is currently dedicated to add navigation and computational abilities to the robot. The front compartment is designed to be replaceable; e.g. various compartments with different computing power or sensory information.

Each joint is actuated by a Faulhaber 2232-006-SR DC micromotor with output power of $11 \mathrm{~W}$ and nominal torque of $10 \mathrm{mNm}$, and with a Faulhaber 22E 69:1 step-down gearbox. With this set-up, we can achieve desired torques at low rotation speeds while keeping the size of the motor reasonably small. A

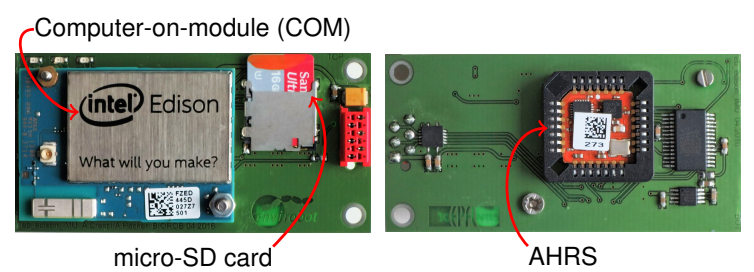

Fig. 4. Linux COM board with (left) Intel Edison on top layer and (right) Xsens MTi-3 AHRS on bottom layer.

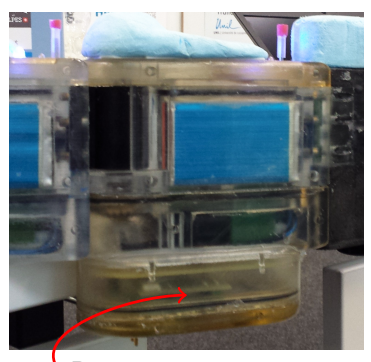

Bottom compartment

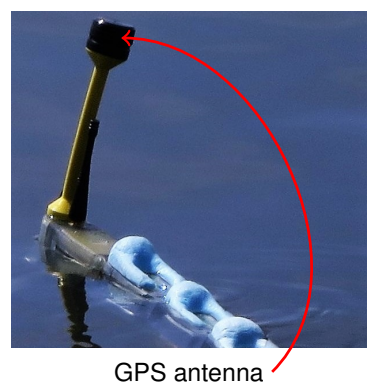

GPS antenna
Fig. 5. Envirobot equipped with (left) temperature and conductivity sensor in the bottom compartment of an active module and (right) GPS antenna on the front compartment.

pair of absolute and relative encoders are used for position and speed control of the joint, using a PD controller implemented on a PIC Microcontrollers (MCU). There are three custom electronic boards in each active module, depicted in Fig. 2. The supply board provides different voltage levels for the electronic components and it also houses the battery charger which charges two 6.66 Wh LiPo batteries when an external power source is connected. The control board has two integrated PIC MCUs, one is dedicated to the low-level motor control [15], and the main PIC is programmed to collect information from local sensors, accept commands from the head module, and even if needed, perform a local sensory feedback control. Furthermore, each module features a connector for sensors, providing power at different voltages, as well access to the local $\mathrm{I}^{2} \mathrm{C}$ bus of the module and to a dedicated CAN bus. This allows connecting a wide variety of commercial and custom sensors, such as temperature, conductivity, $\mathrm{pH}$, and customized bio-sensors developed within the scope of the project. The data collected by the sensors can be read by the head module through the dedicated CAN bus, or (for sensors connected on the $\mathrm{I}^{2} \mathrm{C}$ bus) through the main $\mathrm{CAN}$ bus, via the local PIC18 MCU.

The head unit contains the main low-level controller of the Envirobot. The hardware design is similar to that of active modules, but with the difference that it does not have a motor and its purpose is to provide a higher level of control on top of the controllers of each joint. Simply put, it runs the orchestra of synchronizing the movements of each joint to have a smooth anguilliform swimming, which will be explained with more details in section III. To this effect, it communicates with the other modules through the CAN bus which runs from head to 


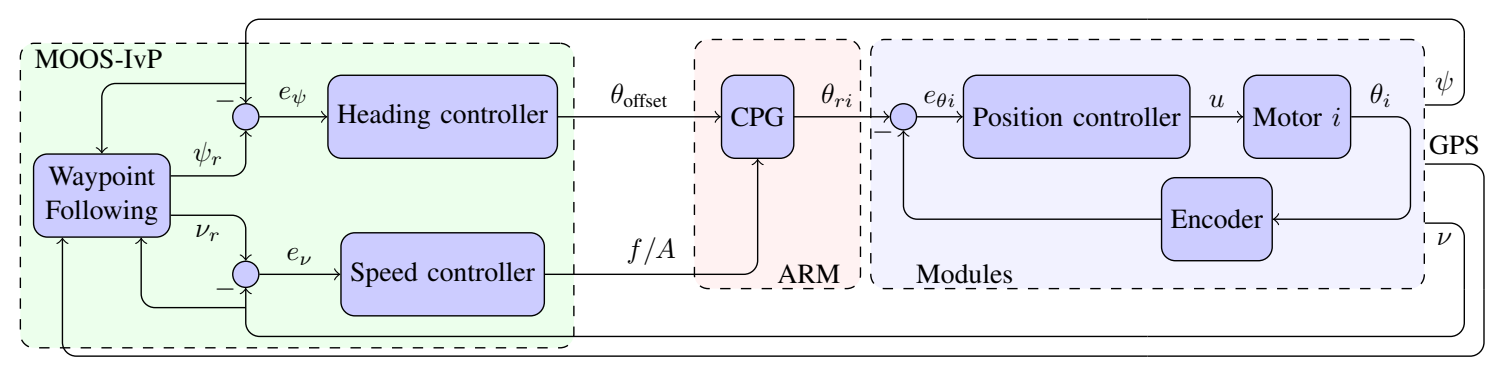

Fig. 6. Control loops in Envirobot are divided to three main levels: (light blue) position controller in each module to control its joint angle, (red) central pattern generator to provide references for each module, and (green) MOOS modules which provide lower level heading and speed control, and and also higher level waypoints following.

tail of the robot. In a sense, it resembles the spinal cord of the robot [16]. Three custom electronic boards of the head unit are depicted in Fig. 3. The supply board is identical to the one used in the other modules. The power board is very similar to the ones used in the other modules but with a few differences. It hosts a connector for a magnetic switch used to turn on/off the robot and a connector providing power and a dedicated UART port for connecting the ARM processor to the Linux computer-on-module (COM). The main control board has an ARM processor which provides sufficient processing power to generate synchronized setpoints for all the joints. Integration of a custom radio transceiver based on a PIC16 MCU and on a nRF905 radio operating on the $868 \mathrm{MHz}$ ISM band, allows bidirectional communication with the ARM processor and also programming it over the RF link.

Currently, the front compartment is dedicated to higher levels of control. It includes an MTi-3 attitude and heading reference system (AHRS) from Xsens, an Edison COM from Intel, a GPS board, and a GPS antenna. We have also planned for future integration of a GPRS module and its antenna for long range communication. Fig. 4 shows the current Linux COM board with the Intel Edison and the AHRS on the top and bottom layer, respectively.

\section{Software Architecture}

In the current structure we have chosen a cascade control strategy. At a very low-level, each module has its own position controller to control its joint angle. This is achieved by the motor control MCU integrated in the module. In one step higher level, a central pattern generator (CPG) [16] in the ARM processor of the head unit provides references for each module, which are received by the the main MCU of each module over the CAN bus connecting all the modules together. We use three parameters of the CPG to control forward speed and heading of the robot. Amplitude of osculation $(A)$ and/or frequency of oscillation $(f)$ has a direct impact on propulsion [17] and are used for forward speed control. On the other hand, the turning offset $\left(\theta_{\text {offset }}\right)$ parameter bends the traveling wave along the robot to a desired direction, which is indeed used for heading control of the robot [18].

In order to develop higher levels of control to accomplish more complicated tasks (e.g., waypoint following, path following, autonomous-navigation, and any other high level

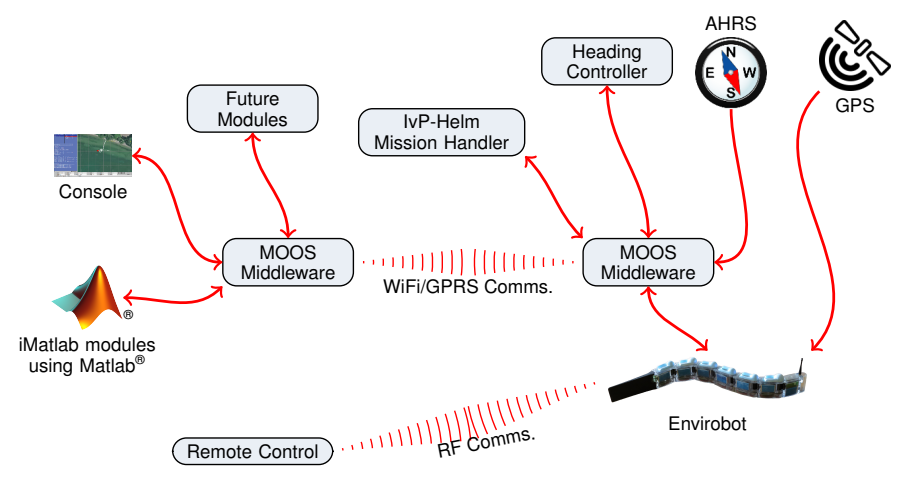

Fig. 7. Software Architecture in Envirobot based on MOOS- middleware.

controller which could be desirable in the future) we use MOOS [19] which is a cross-platform middleware for robotics research. Further, we used IvP-Helm [19] (a set of open-source modules for providing autonomy on robotic platforms running MOOS middleware) to handle missions such as waypoint tracking and path following. Fig. 6 depicts different levels of control implemented in Envirobot.

We use two MOOS middlewares, one on the Envirobot and one on the base-station computer running the console. Any new feature can be added to the platform by defining a new software module interrogating with MOOS middleware. These software modules can be developed in Matlab for fast prototyping, running on the console PC. After further development, extensive tests, and module stabilization, they will be implemented on Envirobot, increasing its autonomous capabilities. An overall software architecture of Envirobot is shown in Fig. 7. Notice that the remote control uses RF for communication and it works independent of the MOOS-IvP. It is only used in the case of emergency to remotely operate the robot and manually override commands to drive the robot to a safe zone.

\section{EXPERIMENTS AND PRELIMINARY RESULTS}

In this section we present some preliminary results and experiments done with Envirobot to test the added navigation and control strategies. Notice that performance of the lowlevel position controller and CPG are previously reported in 


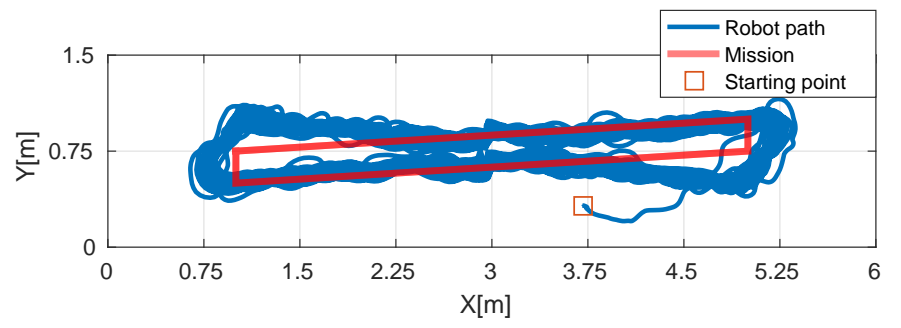

Fig. 8. Path following result of AmphiBot III in the indoor swimming pool.

[7], [8], [15], [17], [20]. Further, in the current state, we only use fixed amplitude and frequency of oscillation, which results in a constant speed if there are no external disturbances, such as currents or waves. Thus, by choosing a reasonably high frequency and amplitude, the robot will be able to face small currents/waves. This is a common practice for autonomous marine/aerial vehicles, which results in less stress on the actuators. This decision is justified due to the fact that, in the current state, the missions are not time dependent; e.g. Trajectory following is an example of time dependent mission which requires an speed controller on the vehicle. In the case of need for a speed controller, one can be simply designed by the GPS speed feedback and actuation on frequency/amplitude of oscillators in the robot. Moreover, the heading controller is extensively discussed and tested in [18]. In this work, we focus on the integration of all the controllers and mainly on waypoint following, which is a key feature to add more functionalities to the robot.

First tests were done in an indoor swimming pool, which is a controlled environment. The pool has a dimension of $1.5 \mathrm{~m}$ by $6.0 \mathrm{~m}$ and depth of $40 \mathrm{~cm}$, just enough for the robot to swim freely. Localization in the indoor swimming pool is achieved by tracking an LED on the head of the robot using existing dual camera tracking system. The tracking system is calibrated such that it gives an accuracy of less than $1 \mathrm{~cm}$. Live connection to indoor tracking system is implemented in a separate MOOS module to replace the positioning data obtained by the GPS. Thus, in terms of data flow, it is equivalent to an outdoor test with availability of GPS. It is a full system integration check before taking the robot outdoors.

Due to the small dimensions of the pool compared to the robot length, autonomous path following using active waypoint following was tested with AmphiBot III, consisting of 7 active modules, which has almost the same hardware and software design but with it a smaller footprint. The indoor path following mission (shown by red track in Fig. 8) is planned such that the robot follows a parallelogram in the pool. The robot starts with a fully charged battery and repeats the mission until the batteries are drained. After fine tuning of the CPG parameters for a reasonably smooth aquatic locomotion, the result was a consistent path following behavior of about 40 rounds in the pool with traveled distance of $430 \mathrm{~m}$, average speed $0.43 \mathrm{~m} / \mathrm{s}$, and total running time 16'50", depicted in Fig. 8 . Note that the small jump (less than $3 \mathrm{~cm}$ ) in the middle of

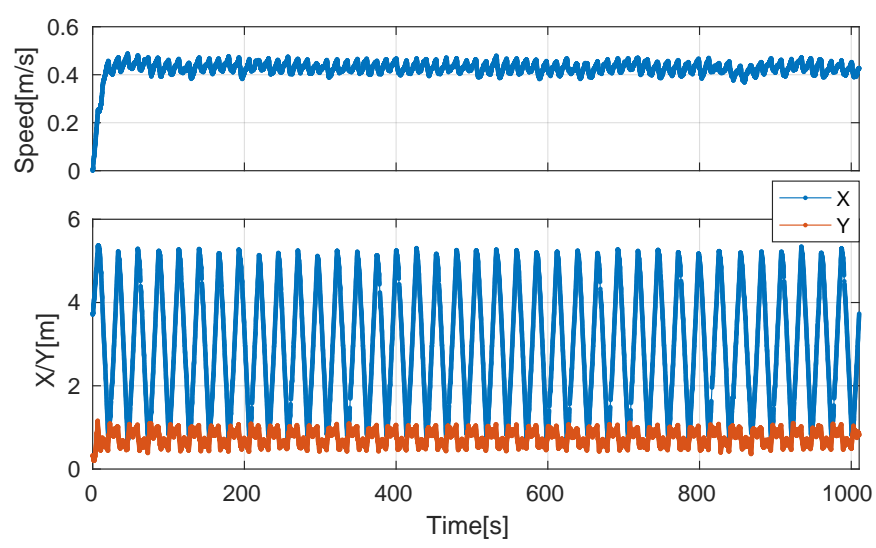

Fig. 9. Position $(\mathrm{X} / \mathrm{Y})$ and speed of AmphiBot III during the path following mission in the indoor swimming pool.

the pool is due to a miscalibration between the two cameras when the tracking system switches from one camera to the next.

The first successful outdoor wet test of Envirobot was done in May 2015 in Lake Geneva, St-Sulpice, EPFL-UNIL sport center (Fig. 10), and was followed by software integration, GPS stability, RF range of communication, and mission control tests in August 2015, in the same location. In both the tests, Envirobot had 6 active modules to perform a natural aquatic locomotion. The results of one of the missions, using only partial battery power, showed traveled distance of $302 \mathrm{~m}$, average speed of $0.87 \mathrm{~m} / \mathrm{s}$, and total running time of $5^{\prime} 48^{\prime \prime}$. CPG oscillation parameters were set to frequency $1.25 \mathrm{~Hz}$, amplitude $35 \mathrm{deg}$, and a complete traveling wave along the body. The chosen parameters are somewhat below the physical constraints of the actuators, which are a frequency of $2 \mathrm{~Hz}$ and an amplitude of $55 \mathrm{deg}$. Thus, by extending the robot to seven active modules and at the same time increasing the oscillation frequency and amplitude, we are expecting to get to a maximum speed of more than $1.5 \mathrm{~m} / \mathrm{s}$, planned to be tested later in the summer 2016.

In August 2016, a series of successful tests were accomplished in Lake Geneva. The main focus of the missions were to put the waypoint following of the robot into a practical test. Fig. 11 shows result of a waypoint following mission (shown in red), which dictates Envirobot to move west for 50 meters and back. The mission was repeated for 7 legs to see repeatability of the result. Since some of the modules were under repair, we used Envirobot with only 3 active modules, oscillation frequency of $1.0 \mathrm{~Hz}$, oscillation amplitude of $30 \mathrm{deg}$, and one third of the traveling wave along the body. Inaccuracy in the GPS track is due to the fact that Envirobot is equipped with a commercial GPS receiver (Maestro A2200A) with an accuracy of 2.5 meters. Furthermore, short length of the robot makes it prone to deviation from the original path, due to frequent waves in the lake. The robot had an average speed of $0.52 \mathrm{~m} / \mathrm{s}$ which is a reasonable speed given the short length of the robot used during the test. Fig. 12 depicts Envirobot velocity, easting, and northing along the 


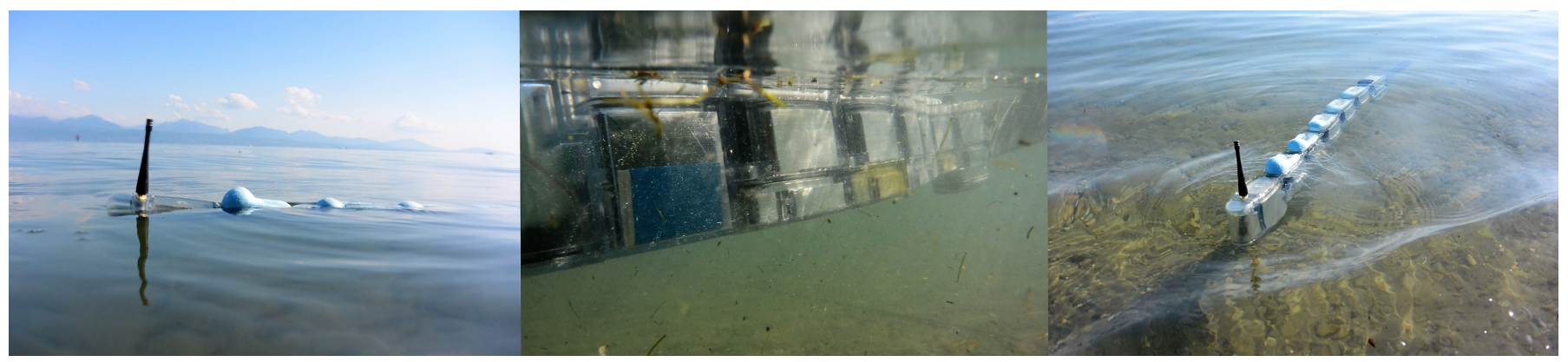

Fig. 10. Snapshots of Envirobot's first wet test in Lake Geneva.

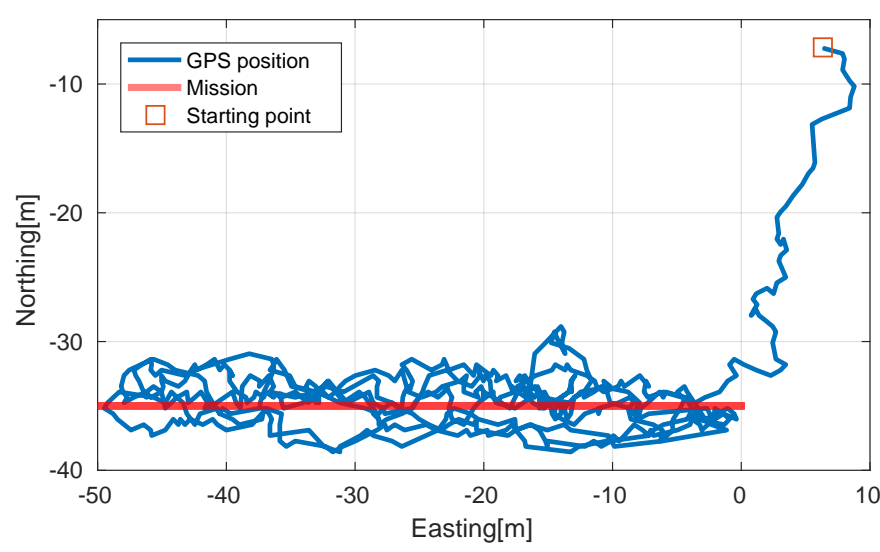

Fig. 11. GPS track of the Envirobot (blue track) starting from $\square$ and following the waypoint following mission (red line) to go 50 meters to the west and back.
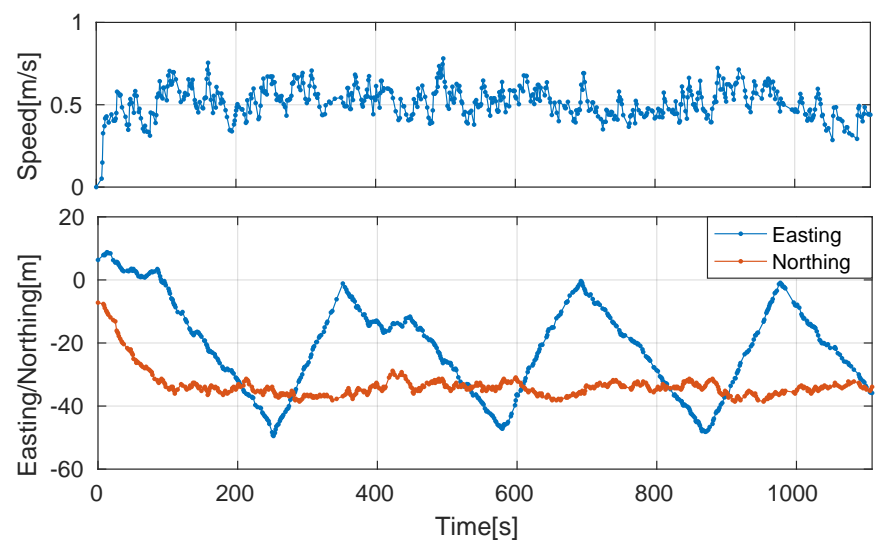

Fig. 12. Easting, northing, and speed of Envirobot during the waypoint following mission.

mission time.

\section{CONCLUSIONS}

With the current progress on the integration of a Linux COM and an AHRS system on Envirobot, several experimental tests are planned in the scope of Envirobot project. Future experimental tests include going to waypoint for water sample acquisition and autonomous surveying for mapping a predefined area with integrated sensors, such as conductivity, temperature, $\mathrm{pH}$, and/or biological sensors. We also plan to conduct endurance tests of Envirobot. Last but not least is implementation and execution of autonomous pollution source seeking on Envirobot to validate the theoretical results obtained in simulations.

\section{ACKNOWLEDGMENT}

The authors would like to thank André Guignard for mechanical design and developing of Envirobot and AmphiBot III, and Howard Li, Mehmet Mutlu, and Rahi Patel for their great help with the experiments in the lake.

\section{REFERENCES}

[1] R. Du, Z. Li, K. Youcef-Toumi, and P. V. y Alvarado, Eds., Robot Fish Springer Berlin Heidelberg, 2015.

[2] D. S. Barrett, "Propulsive efficiency of robotuna," Ph.D. dissertation, Massachusetts Institute of Technology, 1988.

[3] K. A. McIsaac and J. P. Ostrowski, "Experiments in closed-loop control for an underwater eel-like robot," in IEEE International Conference on Robotics and Automation, 2002.

[4] A. Westphal, N. Rulkov, J. Ayers, D. Brady, and M. Hunt, "Controlling a lamprey-based robot with an electronic nervous system," Smart Structures and Systems, vol. 8, no. 1, pp. 39-52, July 2011.

[5] C. Stefanini, S. Orofino, L. Manfredi, S. Mintchev, S. Marrazza, T. Assaf, L. Capantini, E. Sinibaldi, S. Grillner, P. Wallén, and P. Dario, "A novel autonomous, bioinspired swimming robot developed by neuroscientists and bioengineers," Bioinspiration \& Biomimetics, vol. 7, no. 2, May 2012.

[6] P. Liljeback, O. Stavdahl, K. Y. Pettersen, and J. T. Gravdahl, "Mamba - A waterproof snake robot with tactile sensing," in IEEE/RSJ International Conference on Intelligent Robots and Systems. Institute of Electrical \& Electronics Engineers (IEEE), September 2014.

[7] A. Crespi, A. Badertscher, A. Guignard, and A. J. Ijspeert, "Amphibot I: An amphibious snake-like robot," Robotics and Autonomous Systems, vol. 50, no. 4, pp. 163-175, 2005.

[8] A. Crespi and A. J. Ijspeert, "Amphibot II: An amphibious snake robot that crawls and swims using a central pattern generator," in International conference on climbing and walking robots (CLAWAR 2006), 2006, pp. 19-27.

[9] M. Porez, F. Boyer, and A. J. Ijspeert, "Improved lighthill fish swimming model for bio-inspired robots: Modeling, computational aspects and experimental comparisons," The International Journal of Robotics Research, vol. 33, no. 10, pp. 1322-1341, 2014.

[10] Envirobot Project. (2013-2017). [Online]. Available: http://wp.unil.ch/envirobot/

[11] M. Vergassola, E. Villermaux, and B. I. Shraiman, "Infotaxis as a strategy for searching without gradients," Nature, vol. 445, no. 7126, pp. 406-409, January 2007.

[12] B. Bayat, N. Crasta, H. Li, and A. Ijspeert, "Optimal search strategies for pollutant source localization," in submitted to: IEEE/RSJ International Conference on Intelligent Robots and Systems (IROS), 2016.

[13] G. van den Thillart, S. Dufour, and J. C. Rankin, Eds., Spawning Migration of the European Eel. Springer Nature, 2009. 
[14] E. D. Tytell and G. V. Lauder, "The hydrodynamics of eel swimming," Journal of Experimental Biology, vol. 207, no. 11, pp. 1825-1841, 2004

[15] D. Bär, "The ASL I ${ }^{2}$ C motor control module," ASL, Ecole polytechnique fédérale de Lausanne., Tech. Rep., 2013.

[16] A. J. Ijspeert, "Central pattern generators for locomotion control in animals and robots: A review," Neural Networks, vol. 21, no. 4, pp. 642-653, May 2008.

[17] A. Crespi and A. Ijspeert, "Online optimization of swimming and crawling in an amphibious snake robot," IEEE Transactions on Robotics, vol. 24, no. 1, pp. 75-87, Feb 2008.

[18] Y. Morel, A. J. Ijspeert, and A. Leonessa, "Indirect, non-adaptive control of a class of nonlinear uncertain systems with applications to motion control of swimming robots," in ASME Annual Dynamic Systems and Control Conference joint with the JSME Motion and Vibration Conference. Amer Soc Mechanical Engineers, 2013, pp. 179-186.

[19] M. R. Benjamin, H. Schmidt, P. M. Newman, and J. J. Leonard, "Nested autonomy for unmanned marine vehicles with MOOS-IvP," Journal of Field Robotics, vol. 27, no. 6, pp. 834-875, 2010.

[20] A. Crespi, A. Badertscher, A. Guignard, and A. Ijspeert, "Swimming and crawling with an amphibious snake robot," in IEEE International Conference on Robotics and Automation (ICRA), 2005, pp. 3035-3039. 